\title{
On The Evolution of LTE to LTE-Advanced
}

\author{
A. A. Oudah ${ }^{a}$, T. A. Rahman ${ }^{a}$, N. Seman ${ }^{\text {a }}$ \\ ${ }^{a}$ Faculty of Electrical Engineering, Universiti Teknologi Malaysia, 81310 UTM Johor Bahru \\ *Corresponding author: arshedwireless@yahoo.com
}

\section{Article history}

Received: 8 March 2012

Received in revised form: 10 April

2012

Accepted: 18 July 2012

\section{Graphical abstract}

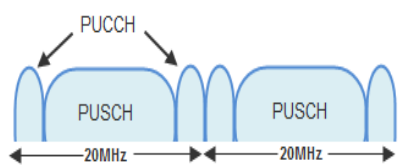

\section{Abstract}

This paper addresses the main features of the transition from the Long Term Evolution standard (LTE) to its successor Long Term Evolution-Advanced (LTE-Advanced). As the process of only creating LTEadvanced specifications alone has taken several years and included thousands of temporary documents, the output thus would be several volumes of specifications. A single paper of this length must therefore choose its contents wisely if it has to do more than just scratching the surface of such a complex standard..

Keywords: Long Term Evolution (LTE); Long Term Evolution Advanced (LTE-A), Multiple-InputMultiple-Output (MIMO); Bandwidth Aggregation; Coordinated Multi-Point (CoMP); Relaying

(c) 2012 Penerbit UTM Press. All rights reserved.

\subsection{INTRODUCTION}

Following the leap from the $2^{\text {nd }}$ Generation $(2 \mathrm{G})$ to the $3^{\text {rd }}$ Generation $(3 \mathrm{G})$ in wireless mobile systems, the International Telecommunication Union (ITU) has come up with the technical requirements of its new evolutionary standard IMT-Advanced [1$11]$.

Historically, ITU is the key player in the whole wireless standardization process. It is the body behind the "G" in all new emerging wireless standards, that is; the $2 \mathrm{G}$, the $3 \mathrm{G}$, and the upcoming 4G [17]. Accordingly, the official name for $3 \mathrm{G}$ is International Mobile Telecommunications-2000 (IMT-2000) while for the $4 \mathrm{G}$ is known as IMT-Advanced [18]. Remarkably, these are not standards as such, they are simply frameworks, and within those frameworks, different bodies submit different candidate technologies. For the third generation, there were five candidate technologies until Worldwide Interoperability for Microwave Access (WiMAX) was added [19]. Up until December 2010 , it appeared there are only two candidate technologies for IMT-Advanced, i.e. the LTE-Advanced and its rival the $802.16 \mathrm{~m}$ standard. In a remarkable turn of events, however, ITU has recently redefined its $4 \mathrm{G}$ to include LTE, WiMAX, and HSPA+[18], [20]. Clearly, LTE standard was, for years, considered as $3.9 \mathrm{G}$ technology and by no means met the 4G targets previously stipulated by ITU [15].

Not surprisingly, this new standard aims at breaking new grounds in extremely challenging spectral efficiency demands that should definitely outperform its predecessors of wireless standards. Average downlink data rates of $100 \mathrm{Mbit} / \mathrm{s}$ in the wide area network and up to $1 \mathrm{Gbit} / \mathrm{s}$ for local access or low mobility scenarios are being the most challenging ones [12-16].
Another key point is that the IMT family members both share the same spectrum, and so there is no $4 \mathrm{G}$ spectrum. There is IMT spectrum, and it is available for $3 \mathrm{G}$ and $4 \mathrm{G}$ technologies. Furthermore, Mobile WiMAX and Ultra mobile broadband (UMB) share, to a certain level, the same radio-interface attributes for those of LTE given in Table 1 [12], [15]. All the three systems, namely; mobile WiMAX, UMB, and LTE, support flexible bandwidths, FDD/TDD duplexing, OFDMA in the downlink and MIMO schemes. However, there are a few differences among them. For instance, the uplink in LTE is based on SC-FDMA compared to OFDMA in Mobile WiMAX and UMB. The performance of the three systems is therefore expected to be similar with minor differences [21-22].

\subsection{LTE SYSTEM OVERVIEW}

In order to meet the non-stop traffic growth demands, extensive efforts have been made in the 3G Partnership Project (3GPP) to develop a new standard for the evolution of 3GPP's Universal Mobile Telephone System (UMTS) towards a packet-optimized system referred to as Long-Term Evolution (LTE). The project, which started in November 2004, featured specifications for a new radio-access technology revolutionized for higher data rates, low latency and greater spectral efficiency [15]. The spectral efficiency target for the LTE system is 3 to 4 times higher than the current High Speed Packet Access (HSPA) system [15]. These challenging spectral efficiency targets require pushing the technology envelope by employing advanced air-interface techniques such as low Peak-to-Average Power Ratio (PAPR), orthogonal uplink multiple access based on Single-Carrier 
Frequency Division Multiple Access (SC-FDMA), multi-antenna technologies, inter-cell interference mitigation techniques, low latency channel structure and Single-Frequency Network (SFN) broadcast to determine LTE [12].

Table 1 Main LTE air interface elements ${ }^{\text {a }}$

\begin{tabular}{|c|c|}
\hline Bandwidth & 1.25-20 MHz \\
\hline Duplexing & FDD, TDD, half-duplex FDD \\
\hline Mobility & $350 \mathrm{Km} / \mathrm{h}$ \\
\hline $\begin{array}{l}\text { Multiple } \\
\text { access }\end{array}$ & $\begin{array}{l}\text { Downlink:OFDMA } \\
\text { Uplink: SC-FDMA }\end{array}$ \\
\hline MIMO & $\begin{array}{c}\text { Downlink: } 2 \times 2,4 \times 2,4 \times 4 \\
\text { Uplink: } 1 \times 2,1 \times 4\end{array}$ \\
\hline $\begin{array}{l}\text { Peak data } \\
\text { rate in } \\
20 \mathrm{MHz}\end{array}$ & $\begin{array}{c}\text { Downlink: } 173 \text { and } 326 \mathrm{Mb} / \mathrm{s} \text { for } 2 \times 2 \\
\text { and } 4 \times 4 \mathrm{MIMO} \text {, respectively. } \\
\text { Uplink: } 86 \mathrm{Mb} / \mathrm{s} \text { with } 1 \times 2 \text { antenna } \\
\text { configuration }\end{array}$ \\
\hline Modulation & QPSK, 16-QAM and 64-QAM \\
\hline $\begin{array}{l}\text { Channel } \\
\text { Coding }\end{array}$ & Turbo code \\
\hline $\begin{array}{l}\text { Other } \\
\text { Techniques }\end{array}$ & $\begin{array}{c}\text { Channel sensitive scheduling, link } \\
\text { adaptation, power control, ICIC and } \\
\text { hubrid ARK }\end{array}$ \\
\hline
\end{tabular}

Remarkably, in the standards development phase, the proposals go through extensive scrutiny with multiple sources evaluating and simulating the proposed technologies from system performance improvement and implementation complexity perspectives [23]. Therefore, only the highest-quality proposals and ideas finally get counted in the standard. The air-interface related elements of the LTE system are summarized in Table 1. The system supports flexible bandwidths, offered by Orthogonal Frequency Division Multiple Access (OFDMA) and SC-FDMA access schemes. In addition to Frequency Division Duplexing (FDD) and Time Division Duplexing (TDD), Half-Duplex FDD (HD-FDD) is allowed to support low cost User Equipment (UE) [23], [24]. Unlike FDD, in HD-FDD operation a UE is not required to transmit and receive at the same time, thus avoiding the need for a costly duplexer in the UE.

The system is primarily optimized for low speeds up to 15 $\mathrm{km} / \mathrm{h}$. However, the system specifications allow mobility support in excess of $350 \mathrm{~km} / \mathrm{h}$ at the cost of some performance degradation [25]. The uplink access is based on SC-FDMA that promises increased uplink coverage due to low PAPR relative to OFDMA. The system supports downlink peak data rates of 326 $\mathrm{Mb} / \mathrm{s}$ with " $4 \times 4$ "multiple-input multiple-output (MIMO) within $20 \mathrm{MHz}$ bandwidth [24]-[26]. Since uplink MIMO is not employed in the first release of the LTE standard, the uplink peak data rates are limited to $86 \mathrm{Mb} / \mathrm{s}$ within $20 \mathrm{MHz}$ bandwidth. Similar improvements are observed in cell-edge throughput while maintaining same-site locations as deployed for HSPA.

In terms of latency, the LTE radio-interface and network provide capabilities for less than $10 \mathrm{~ms}$ latency for the transmission of a packet from the network to the UE [25].

\subsection{LTE-ADVANCED SOLUTION PROPOSALS}

This section gives an overview on LTE-Advanced solution proposals. The solution proposals come in five groups: Bandwidth aggregation, Enhanced uplink multiple access, Higher order MIMO, Coordinated Multipoint (CoMP) and Relaying.

\subsection{Bandwidth Aggregation}

With a goal of $1 \mathrm{Gbit} / \mathrm{s}$, it is clear that this will not be met out of existing channel bandwidths. At the moment, LTE supports up to $20 \mathrm{MHz}$, and it is understood that the ability to improve spectral efficiency much beyond the current LTE performances is very much unlikely, and therefore the only way to achieve that higher data rates is to increase the channel bandwidth. 40 and $100 \mathrm{MHz}$ have been set as the lower and upper bandwidths limits for both LTE-Advanced and IMT-Advanced, respectively [27]. The problem with $100 \mathrm{MHz}$ is that the spectrum is scarce, and 100 $\mathrm{MHz}$ of adjacent spectrum is simply not available in most cases. Hence, to solve this problem, ITU has decided to do bandwidth aggregation between different bands. This means that spectrum from one band can be added to spectrum from another band. Figure 1 shows a contiguous aggregation, where two $20 \mathrm{MHz}$ channels have been taken and put side by side.

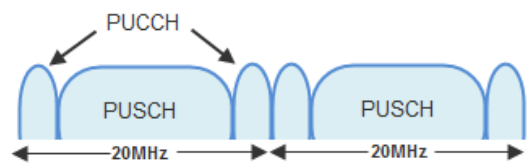

Figure 1 Contiguous aggregation of two $20 \mathrm{MHz}$ uplink component carriers $^{\text {a }}$

In this case, this can be done by means of a single transceiver. But in the case where additional spectrum is not adjacent to the channel in use, then we are talking about spectrum aggregation among different bands which require multiple transceivers. The terminology used to describe this is called a component carrier, which is currently one of the six bandwidths defined for LTE. However, it is possible to aggregate different numbers of component carriers, but the maximum size of a component carrier will be limited to 110 resource blocks, which corresponds to $19.8 \mathrm{MHz}$ for LTE. This has not been confirmed yet and it may be that the existing $18 \mathrm{MHz}$ maximum of 100 resource blocks will remain the maximum for LTE-Advanced.

Clearly, there are a lot of spectra around, namely: 22 FDD frequency bands for LTE as well as a number of bands for TDD [27], [28]. This means there are a lot of possibilities for aggregating different bands. However, the challenge is which bands should be picked. Since the answer as to which bands should be aggregated depends largely on the geography of the deployment.

To help with this problem, the committee has identified twelve scenarios [29] which are most likely to be deployed, and the challenge here is endeavoring to Figure out what are the requirements for issues like spurious emissions, maximum power and all the issues that emanate from combining different radio frequencies into one device.

\subsection{Enhanced Uplink Multiple Access}

The next major feature is the enhancement to the uplink access scheme. LTE is based on SC-FDMA, a smart system that has a lot of the flexible features inherent to Orthogonal Frequency Division 
Multiplexing (OFDM) plus the low PAPR of single carrier systems.

Figure 2 shows an example of various SC-FDMA schemes. Here we have, for instance, a $20 \mathrm{MHz}$ channel, and we see at the edge of the channel the control channel (PUCCH), which operates one resource block, or $180 \mathrm{KHz}$. Somewhere within the bandwidth is the shared channel (PUSCH) which uses the SCFDMA modulation.

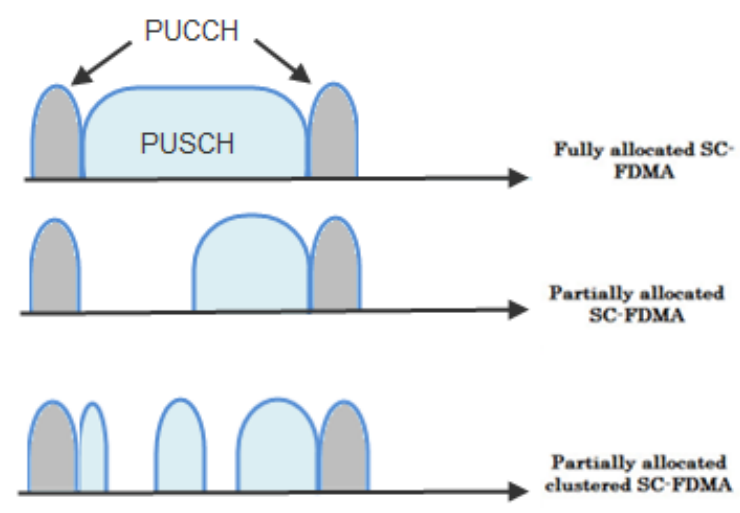

Figure 2 Various SC-FDMA schemes

And there are three possibilities here; the first two graphs from the upper side are inherent to LTE. However, the new technique that has come in with LTE-Advanced is called clustered SC- FDMA, where the spectrum is not fully occupied as indicated at the bottom of Figure 2. The reason is to provide more flexibility in the uplink when the channel is frequency selective. Notably, the problem with SC-FDMA is picking a contiguous block of allocation. Thus, if a channel displays a certain variation in performance across frequency, then, decision should be made about where to allocate the signal.

The advantage of the clustered approach is that the same allocation in terms of bandwidth can be taken and split up into different slices within the overall channel bandwidth, and this is where the concept of clustering comes in. It has a slight degradation on PAPR performance, but it is significantly better than the alternative, which is to use pure OFDM, as in other systems like WiMAX. Pure OFDM allows the highest flexibility in the uplink, but it also suffers from very high PAPR. So the concept of clustered SC-FDMA is an excellent tradeoff between OFDM flexibility and low PAPR of the original SC-FDMA.

\subsection{Multiple-Input Multiple-Output (MIMO)}

The next major feature of LTE-Advanced is higher order MIMO transmission. Historically, the following limits were established by Release- 8 LTE [25]: the downlink has a maximum of four layers MIMO of transmission, while the uplink has a maximum of one - for -one mobile. So this together with the fact that the UE has received diversity means we could support " $4 \times 2$ " MIMO in the downlink and in the uplink there is no MIMO as such from a single mobile device. Now with LTE-Advanced, the situation is considerably different. There is general consensus of supporting up to eight streams in the downlink with eight receivers in the UE. This will give a possibility of " $8 \times 8$ " MIMO in the downlink. And in the uplink, the UE is capable of supporting up to four transmitters, thereby offering a possibility of up to " $4 \times 4$ " transmissions. The additional antennas can also be used, say, for beamforming and the overall goal is to increase the data rates coverage capacity of the cell.
So far, they have not been commercially available, although there are a lot of challenges with higher order MIMO; in particular, how to deal with the actual transmission at the base station terminals and issues like tower-mounted radio heads. Furthermore, the power consumption is another issue, both at the base station and the UE. Products themselves will become more complex and costlier. Finally, there is a physical space which the antennas take up and this is a particular issue for mobile devices, although products like portable computers, for instance, would be able to take on larger numbers of antennas.

\subsection{Coordinated Multi-Point (CoMP)}

In traditional MIMO systems, shown in Figure 3, there is a transmitting unit in which a base station with more than one antenna going through a channel to a receiving unit having more than one receiver. On the other hand, with coordinated multipoint, the difference is that at the transmitting end the two entities are not necessarily physically located, although they are connected with some form of a high-speed data connection. Accordingly, in the downlink, this allows for coordinated scheduling and beamforming from two different locations. This implies that the system is not fully utilized as the data required to be transmitted to the UE only needs to be present at one of the serving cells. That is, some amount of partial coordination has taken place. However, if we go for coherent combination, also known as cooperative MIMO, then it is possible to do more advanced transmission whereby the data which is being transmitted to the UE is coming from both locations, and it is coordinated at the UE with pre-coding techniques in order to maximize the signal-to-noise ratio (SNR). The challenge of this approach is that there is need to have a high-speed symbol level data communication between both transmitting units, as indicated by the vertical black arrow in Figure3.

Within LTE, there is the concept of the "X2" interface, which is a mesh-based interface between the base stations. By this mechanism, this physical link is likely to be the one to be used for sharing the base band data. One way of looking into coherent combining is a bit like soft combining or soft handover; which is widely applied in Code Division Multiple Access (CDMA) systems, except that the data being transmitted is not identical from both base stations. They are two different data streams which are then coordinated in such a way to allow the mobile device to receive both simultaneously. In the uplink, the use of coordination between the base stations is less advanced because when there are more than one device in different places, there will be no realistic mechanism for sharing data between the two transmitting devices. Therefore, in the uplink, the concept is more limited to the earlier version of the downlink, which is to coordinate on scheduling.

\subsection{Relaying}

A relaying its simplest form is otherwise referred to as a repeater; a device which receives the transmissions within the channel of interest at its input, amplifies them and then retransmits to the local area. It is also used for improving the coverage, although with no substantial capacity improvement. Recently, the concept of relaying is to take this a stage further by decoding the transmissions which is fed into the cell of interest and instead of only retransmitting the amplified inputs to the rest of the cell or the targeted area, it would selectively retransmit a portion of the transmission. Relaying is possible at different layers in the protocol. The most advanced one being layer three relaying, in which the relay node would pick out only the traffic for the mobile device within its vicinity and retransmit the signal. This is 
carried out without transmitting any other signals for mobile devices which may be in the macrocell but are not associated with the relay node. Therefore, this makes a kind of selective repeater where the problem of adding interference to the network is reduced on the downlink. On the other hand, in the uplink the relay node is not connected to the network via some form of cabled backhaul, which is the case with the macro cell. Hence, it is possible to deploy a relay node at some distance from the macrocell or serving node without having to deal with any cabling problems in order to get the backhaul.
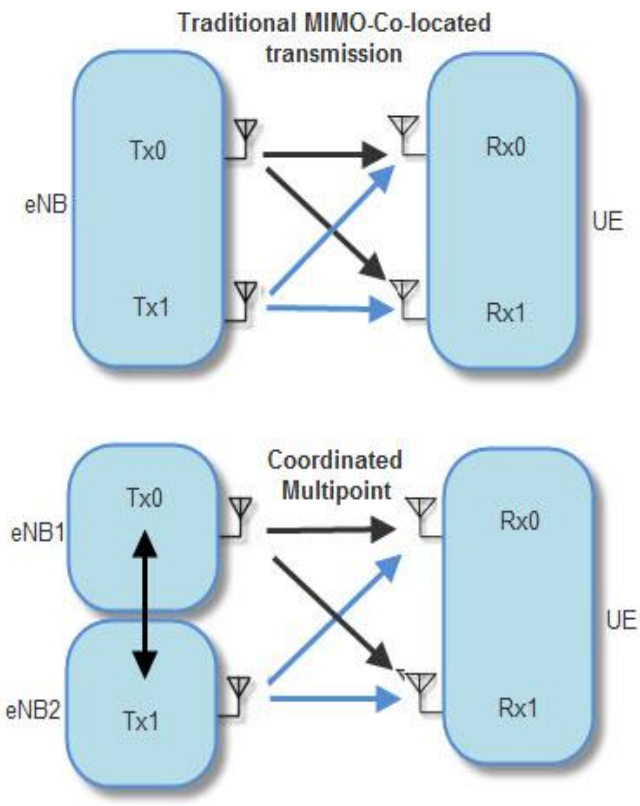

Figure 3 The differences between traditional MIMO and CoMP

For instance, in a situation where coverage is sought in, say, some remote locations down a valley, it is possible to employ a multi-hop relay whereby a signal will be sent from the serving cell to the relay node down to the UE. Accordingly, the signal coming from the UE would be transmitted up to the relay node, which is now in the form of backhaul, which would transmit this signal back to the base station using the same channel as used for the downlink in a TDD system, or the complementary channel in an FDD system. The reason it is possible to do this in an OFDM system is that it is possible to split the channel into different parts. No need to use the whole channel for all transmissions. Thereby, a cell could allocate half of the uplink resource blocks to relay backhaul traffic and the other half to UEs in the macro network.

This means the OFDM provides a lot of flexibility to do this form of in-channel backhaul, which otherwise would be impossible in a CDMA system unless a new channel is introduced.

There are different ways in which relaying could be used, but they basically fall into a couple of major areas, one is to do selective improvements to coverage. Also there are other aspects of relaying which would appear to provide throughput advantages within the macrocell.

In fact, a lot of work still needs to be done on relaying and there is consensus on how this particular feature will be deployed. In some ways, we could look upon relaying as a more advanced form of repeating where we may have one or two of these types of devices in a macrocell. However, there are other schools of thought which suggest that a macrocell might support hundreds of relay nodes in order to provide much higher level of capacity in such a way that is similar to the concept of Femtocells, except that the whole system will be coordinated from the center.

In general, there is a fact that we are looking at many different types of cells now, from Macro to Pico to Femtocells and recently these relay nodes; and what is happening within the radio environment is a much higher level of hierarchy within the scope of the different base stations. This creates a hierarchical, rather than a homogenous, network where each cell is at the same level in the hierarchy and they are all one big sort of mosaic of coverage, thus leading to the concept of a hierarchical network where we have umbrella types of coverage having much smaller coverage areas with different techniques. This, however, presents some real challenges to the whole radio management. And the subject of radio resource management is a major item which continues to develop as the radio environment becomes more complex.

Heterogeneous network is not an item as such in LTEAdvanced, but the fact that Femtocells will be coming along soon in these relay nodes means that there will be a substantial need to research and develop mechanisms to enable these more complex radio networks to function efficiently. It is worth mentioning here that the key difference between Femtocells and traditional cells is the backhaul and the fact that these devices are not centrally managed. However, most people would tend to think of Femtocells as being smaller versions of Picocells. But if we think of it in terms of backhauling and planning, they are, in fact, extremely different in the way they interact with the network. Also, there are other factors such as cost and the performance expectations, and so on. Femtocells are one of the elements in the heterogeneous network which are being developed in the standards and by the time LTE-Advanced comes along; they will definitely be part of the landscape.

\subsection{PROS AND CONS OF LTE-ADVANCED}

In order to summarize the overall picture of LTE-Advanced, Table 2 shows a list of attributes of the five main features this paper discusses. The table provides answers to the following arising questions: what do these features provide in terms of performance and what is the cost of actually deploying them?

Beginning with bandwidth aggregation, which is a very obvious key player here, it is primarily aimed at peak data rates with no substantial change in spectral efficiency, although we may get some benefits from the fact that a larger instantaneous channel is available to multiple users. Cell edge performance as well as coverage would not change.

However, when it comes to the cost, particularly in the UE, there would be substantial issue in bandwidth aggregation, if it is non-contiguous and the mobile device had to support more than one transceiver, or in the worst case, up to five different transceivers. Clearly, this translates to a significant cost increase. On the network side, it is unlikely that there would be any significant cost change since the base station is typically standalone in terms of different frequency bands. Whereas there would be an increase in overall network complexity, and this is mentioned here, primarily on the UE side.

Looking at enhanced uplink, the clustered SC-FDMA, there is no appreciable change in peak data rates. This is because if the peak data rate is required, a whole channel has to be allocated, and therefore clustering has no meaning. But the intention behind this technique is to take the advantage of the frequency- selective channel; thus, offering a benefit of spectral efficiency, although it is not a major change over what we have today. Similarly, there 
may be some advantages in cell edge performance. However, with regard to overall coverage, it is hard to know whether or not there would be a coverage support.

Table 2 Expected pros and cons of LTE-Advanced system parameters
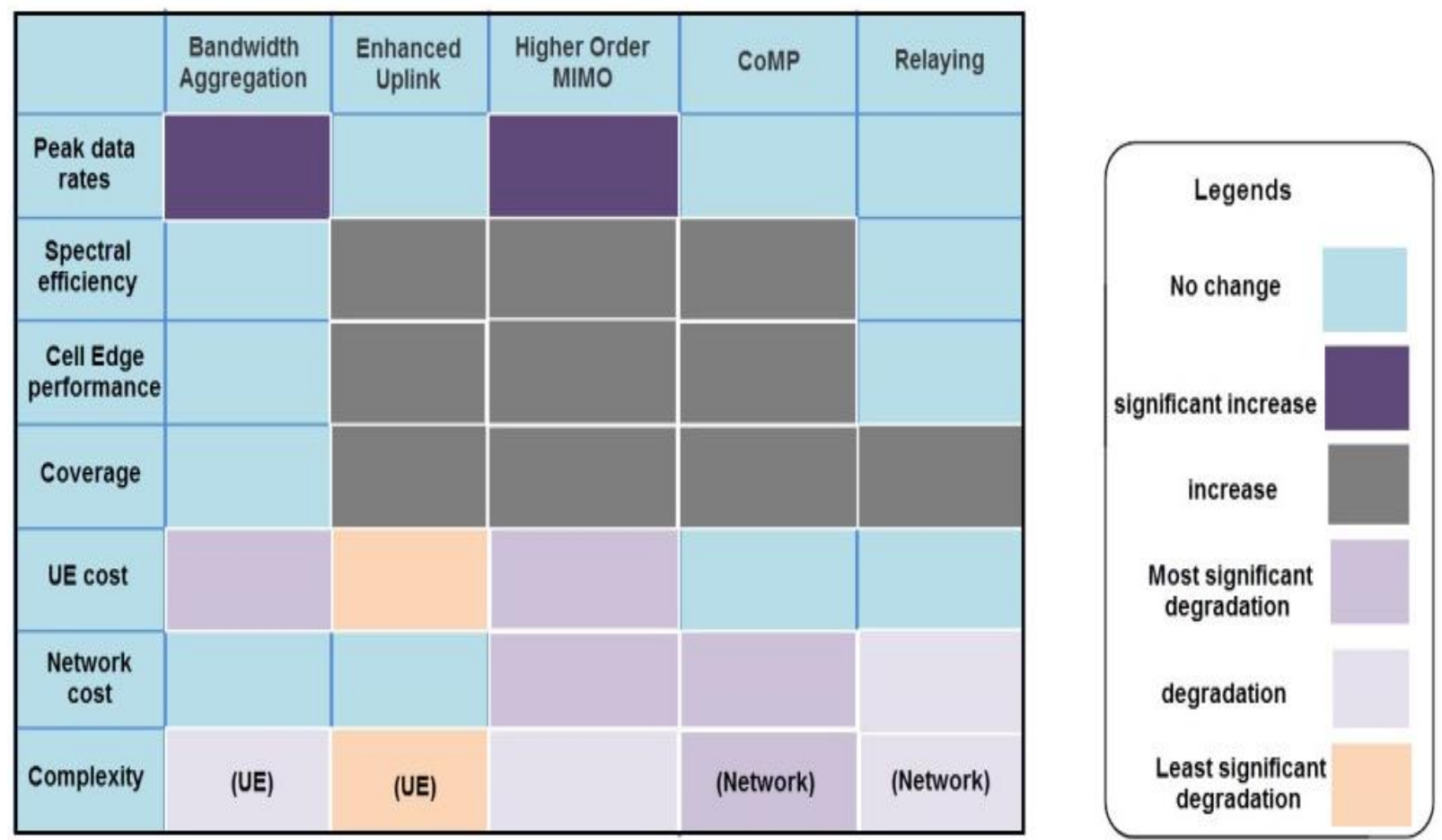

In terms of UE cost, it is unlikely that it would be significant. Concerning network cost, it is uncertain to have any impact and some minor increase in UE complexity. Considering the higher order MIMO, the expectations for peak data rates are driven by some of these " $8 \times 8$ " downlink or " $4 \times 4$ " uplink antenna configurations. Also, there will be benefits in terms of spectral efficiency, cell edge performance and coverage through the different techniques.

MIMO is not a single subject. Notably, in basic LTE, there are seven different transmission modes in the downlink, all varying from traditional type up to closed loop MIMO. With the introduction of more antennas in LTE-Advanced, there are many different ways we could use these antennas depending on the particular radio environment. Hence, it is impractical to attribute a particular benefit to one particular scenario. It very much depends on whether the system is developed to take advantage of a particular scenario. But in general, higher order MIMO should lead to increases in the average in cell edge and coverage performance.

However, when it comes to the cost, clearly in the mobile device if we have to implement multiple transceivers in the UE to support these different streams, there is a big impact in terms of the product cost. Going from one to two and to four transmitters is a big issue. It is interesting to note that LTE, in its basic form, does not support uplink MIMO. It is a single transceiver approach, while LTE-Advanced will be taking advantage of up to four transceivers. Accordingly, there could be a big impact on the cost of the mobile device. On the network side, there would be an increase, though it may not be as noticeable as on the mobile side, because most networks on the base station side already have probably two antennas at the moment and some maybe four. But certainly there would be an increase. And then in the overall complexity of the system, there would be an increase as well. Regarding the coordinated multipoint, it is not likely to have any impact on peak rates, but again, similar to MIMO, there might be expectations on spectral efficiency improvement, cell edge performance and coverage. UE cost, unlikely to have any impact at all, but on the network side, CoMP could be a big issue, and that is primarily because of the need for the high speed backhaul between the different base stations. With regard to complexity, certainly, there will be a major increase in complexity in terms of real time management of all these coordination among the base stations.

Finally, considering relaying, it is unlikely to have any effect on peak rates or efficiency, but some improvements in cell edge and coverage are possible; as those are the main areas that are being targeted by relaying. And no impact, obviously, on the cost of UE, as the UE should view a relay network in the same way as it views the standard network. But, there would be an increase, obviously, in network cost; because the relay nodes need to be deployed. Not the least is the issue of network complexity which is higher than standard networks due to the management of the relay nodes.

\subsection{CONCLUSIONS}

LTE-Advanced is 3GPP's submission to the ITU radio communications sector; IMT-Advanced program. It is important to differentiate between IMT-Advanced, which is the ITU's family of standards, and LTE-Advanced, which is the 3GPP candidate submission. LTE-Advanced clearly is an evolution of LTE, and it is approximately two years behind. In terms of standardization, however, trying to predict the deployment date for LTE-Advanced is much harder, because we are trying to extrapolate from something that is already somewhere in the 
future. However, IMT-Advanced deployment is still several years away whereas deployment of HSPA Evolution (HSPA+) and LTE is already ongoing.

\section{Acknowledgement}

The author(s) would like to thank Professor Dr. Tharek Abdul Rahman-director of wireless communications center and Head of IMT-Advanced group for his guidance and continuous support.

\section{References}

(1) The Global Mobile Suppliers Association (GSA). 2011. Status of the LTE Ecosystem. http://www.gsacom.com/gsm_3g/info_papers.php4.

(2) H. Holma and A. Toskala. 2011. LTE for UMTS: Evolution to LTEAdvanced. John Wiley and Sons.

(3) K.-L. Du and M. N. S. Swamy. 2010. Wireless Communication Systems: From RF Subsystems to $4 G$ Enabling Technologies. Cambridge University Press. 985.

(4) S. Sesia, I. Toufik, and M. Baker. 2011. LTE-The UMTS Long Term Evolution: From Theory to Practice. John Wiley and Sons. 680.

(5) The Global Mobile Suppliers Association (GSA). 2011. Mobile Broadband Fact Sheet http://www.gsacom.com/gsm_3g/info_papers.php4.

(6) A. F. Molisch. 2011. Wireless Communications. John Wiley and Sons. 884.

(7) The Global Mobile Suppliers Association (GSA). 2011. Evolution to LTE Report. http://www.gsacom.com/gsm_3g/info_papers.php4.

(8) 3GPP TS 36.300. 2006. Evolved Universal Terrestrial Radio Access (E-UTRA) and Evolved Universal Terrestrial Radio Access Network (E-UTRAN); Overall description; Stage 2.

(9) 3GPP TS 36.410. 2010. Evolved Universal Terrestrial Radio Access Network (E-UTRAN); S1 general aspects and principles.
(10) A. Ghosh and R. Ratasuk. 2011. Essentials of LTE and LTE-A. Cambridge University Press. 272

(11) The Global mobile Suppliers Association (GSA). 2011. Mobile Broadband Wallchart: 3GPP systems. http://www.gsacom.com/gsm_3g/info_papers.php4.

(12) 3GPP TS 25.201. 2007. Physical layer - general description.

(13) 3GPP TS 36.104. 2009. Evolved Universal Terrestrial Radio Access (E-UTRA); Base Station (BS) radio transmission and reception.

(14) 3GPP TS 36.213. 2009. Evolved Universal Terrestrial Radio Access (E-UTRA); Physical layer procedures.

(15) 3GPP TR 25.913. 2009. Requirements for Evolved UTRA (E-UTRA) and Evolved UTRAN (E-UTRAN)

(16) C. Johnson. 2010. Long Term Evolution IN BULLETS. 1st ed. CreateSpace.

(17) ITU. 2010. ITU paves way for next-generation 4G mobile technologies/ ITU-R IMT-Advanced 4G standards to usher new era of mobile broadband communications. [Online]. Available: http://www.itu.int/net/pressoffice/press_releases/2010/40.aspx.

(18) ITU. 2010. ITU World Radiocommunication Seminar highlights future communication technologies. [Online]. Available: http://www.itu.int/net/pressoffice/press_releases/2010/48.aspx.

(19) WiMAX Forum. 2010. WiMAX Forum® Air Interface Specifications.

(20) S. Yin. 2010. ITU Redefines 4G. Again. pcmag.com. [Online]. Available: http://www.pcmag.com/article2/0,2817,2374564,00.asp.

(21) T. Ali-Yahiya. 2011. Understanding LTE and Its Performance. Springer. 264.

(22) E. Dahlman, S. Parkvall, and J. Sköld. 2011. 4G: LTE/LTE-Advanced for Mobile Broadband. Academic Press. 455.

(23) M. Rumney. 2009. Agilent technologies: LTE and the Evolution to $4 G$ Wireless: Design and Measurement Challenges. 1st ed. Wiley.

(24) F. Khan. 2009. LTE for $4 G$ Mobile Broadband: Air Interface Technologies and Performance. Cambridge University Press. 506.

(25) 3GPP. 2009. Technical Specifications Rel.8.

(26) E. Dahlman, S. Parkvall, and J. Skold. 2011. 4G: LTE/LTE-Advanced for Mobile Broadband. Academic Press.

(27) 3GPP. 2009. TS 25.913: Requirements for Evolved Universal Terrestrials Radio Access Network.

(28) 3GPP. 2009. Study Phase Technical Report TR 36.912 v2.2.0.

(29) 3GPP. 2009. Latest Status report RP-090729. 